\title{
Crosstalk between vitamin $D$ axis, inflammation and host immunity mechanisms: A prospective study
}

\author{
ANDREEA-DANIELA MECA ${ }^{1}$, SIMONA ȘTEFĂNESCU ${ }^{2}$, MARIA BOGDAN $^{1}$, ADINA TURCU-ȘTIOLICĂ ${ }^{3}$, \\ FLOAREA MIMI NIȚU ${ }^{4}$, MARIUS MATEI ${ }^{5}$, RAMONA CIOBOATĂ $\breve{C}^{4}$, \\ ANA MARIA BUGĂ ${ }^{6}$ and CĂTĂLINA-GABRIELA PISOSCHI ${ }^{6}$ \\ ${ }^{1}$ Department of Pharmacology, University of Medicine and Pharmacy of Craiova, 200349 Craiova; ${ }^{2}$ Clinical Laboratory, \\ Clinical Emergency County Hospital, 200642 Craiova; Departments of ${ }^{3}$ Pharmacoeconomics, ${ }^{4}$ Pneumology, \\ ${ }^{5}$ Histology, ${ }^{6}$ Biochemistry, University of Medicine and Pharmacy of Craiova, 200349 Craiova, Romania
}

Received January 15, 2021; Accepted February 16, 2021

DOI: $10.3892 /$ etm.2021.10040

\begin{abstract}
Tuberculosis (TB) remains a public health burden, after many years at attempts for its eradication. Vitamin D (VD) status has been suggested to be related to TB susceptibility because it has the ability to regulate multiple axes of the innate and adaptive host immune response. VD mediates cathelicidin (LL-37) synthesis, a cationic bactericidal peptide, through the expression of vitamin D receptor (VDR). Host innate defense mechanisms include autophagy and apoptosis of alveolar macrophages. The present study aimed to assess the relationship between VD status, inflammation and host defense mechanisms before and after two months of first-line anti-TB pharmacotherapy. The study included newly diagnosed individuals with pulmonary TB without co-morbidities (HIV infection, diabetes, cancer) and without VD supplementation or other therapies interfering with VD serum levels. We measured serum levels of 25-hydroxyvitamin D (25-(OH)-D), the major circulating form of vitamin D, VDR, LL-37, beclin-1 (an autophagy marker) and M30 (an apoptosis biomarker) before and after two months of anti-TB treatment. Individuals presented lower levels of 25-(OH)-D before receiving first-line
\end{abstract}

Correspondence to: Dr Ana Maria Bugă, Department of Biochemistry, University of Medicine and Pharmacy of Craiova, 2-4 Petru Rareș Street, 200349 Craiova, Romania

E-mail: anne_mary07@yahoo.com

Dr Adina Turcu-Știolică, Department of Pharmacoeconomics, University of Medicine and Pharmacy of Craiova, 2-4 Petru Rareș Street, 200349 Craiova, Romania

E-mail: adina.turcu@gmail.com

Abbreviations: M.tb., Mycobacterium tuberculosis; TB, tuberculosis; TLR, Toll-like receptor; VD, vitamin $\mathrm{D}_{3}$ (25-hydroxyvitamin D); VDR, vitamin D receptor; LL-37, cathelicidin; M30, apoptosis biomarker; T0, before anti-tuberculosis treatment; T2, after two months of anti-tuberculosis treatment

Key words: tuberculosis, vitamin $\mathrm{D}_{3}$, vitamin $\mathrm{D}$ receptor, cathelicidin, apoptosis, autophagy
anti-TB treatment (T0) in comparison with its plasmatic levels after two-months of therapy (T2). At T2, patients were divided in two subgroups according the results of sputum-culture conversion. After two-months of therapy, decreased values of LL-37, beclin-1 and M30 were observed in the culture-negative patients compared to the culture-positive patients. Control of anti-TB treatment outcome could be improved by appraisal of VD status and host defense mechanisms such as autophagy and apoptosis.

\section{Introduction}

Controlling infection with Mycobacterium tuberculosis (M.tb.) has become one of the top priorities in the last few years as tuberculosis (TB) remains a global threat $(1,2)$. More than 7 million cases were estimated to be diagnosed worldwide in 2018 , from which $85 \%$ were diagnosed with pulmonary TB (3). Almost 1.5 million deaths were reported in 2018 among both HIV infected and uninfected individuals (2). These facts increase the need to use host-directed immunomodulatory therapies among classical antituberculosis treatment, as susceptibility to infection is determined by both bacteria and host $(1,4)$. Autophagy is an essential intracellular homeostatic proteolytic mechanism which has been recently discussed as an antimicrobial strategy in pulmonary TB $(5,6)$. The specific histopathological hallmark of TB is represented by the granuloma, an immunological environment where infected macrophages induce secretion of inflammatory cytokines and further recruitment of neutrophils, monocytes and lymphocytes (7). Macrophages restrain M.tb. by phago-lysosomal fusion and bacterial degradation to immunogenic polypeptides, therefore preventing dissemination of the bacilli $(7,8)$. The question is if macrophages are more than antigen-presenting cells and immunological barriers (8). Indeed, macrophages possess bactericidal activity, creating a dynamic connection between innate immunity and adaptive host-responses (7). Specific M.tb. recognition membrane-receptors, Toll-like receptors (TLR), lead to cytokines and antimicrobial effector production $(7,8)$. Even more, TLR signals involve $\mathrm{CYP}_{27} \mathrm{~B}_{1}$ stimulation which catalyses hydroxylation of cholecalciferol (vitamin $\mathrm{D}_{3}$ ) into its most active form 1,25-(OH $)_{2}$-cholecalciferol called 
calcitriol (8). Following upregulation of T-cells as an immune adaptive response, the activated vitamin $\mathrm{D}_{3}$ (VD) form binds to specific vitamin D receptors (VDRs), constitutively expressed in immune cells such as macrophages, monocytes, and dendritic cells $(5,8,9)$. Therefore, the role of VD as an essential endogenous immune mediator in pulmonary TB and not just as a maintainer of calcium homeostasis and musculoskeletal health is highlighted $(5,9)$. The overall amount of evidence reporting the anti-inflammatory and immunoregulatory action of the active VD forms suggests a function that might be generally called 'stress-quenching activity' (7-9). VD activates immunological pathways by direct induction of specific antimicrobial peptides, such as cathelicidin (LL-37) and $\beta$-defensins, in phagocytes and by stimulation of autophagic processes, assessed through autophagy-related genes, such as beclin-1 and Atg5 (4,5). Many studies have emphasized the dose-dependent bactericidal activity against M.tb. of both VD and LL-37 $(4,5,9)$ and also the essential role of beclin-1 in phagosome maturation and cellular apoptosis of macrophages $(6,10)$. On the other hand, VD deficiency leads to negative lymphoproliferative response, decreased autophagy, lower regulation of the pathological response and downregulated bacterial clearance (4,6,7). M.tb. is further able to multiplicate and persist at a low metabolic rate in the granuloma, developing into extensive tissue injury, caseous necrosis and cavitary severe forms $(7,8)$.

In the case of persistent infection, as an attempt to eliminate infected immune cells, epithelioid cells of the tuberculous granuloma produce M30 (an epitope of caspase-cleaved cytokeratin 18) (11,12). M30 is a specific apoptosis biomarker, obtained after cleavage of various cellular proteins through caspase activity, that can be measured from plasma through immunochemical methods (11). The programmed cellular death of macrophages could reduce the viability and limit the spread of M.tb, without spilling cellular affected contents into the tissue, and eventually promote host defense by stimulating an adaptive immune response through $\mathrm{T}$ cells $(10,12)$. Yet, the mycobacteria are able to adopt different strategies, yet incompletely known, in order to avoid apoptosis. One of the most relevant is the preferential undergoing necrosis of macrophages, which causes acute inflammation and tissue swelling (13).

In the present study, we assessed serum levels of VD, VDR, LL-37, beclin-1 and M30 before and after two months of anti-TB treatment in order to assess the crosstalk between VD axis, inflammation and host defense mechanisms and to determinate the outcome of first-line anti-TB pharmacotherapy.

\section{Patients and methods}

Study design. A total of 30 active newly diagnosed pulmonary TB patients from 'Victor Babeș' Clinical Hospital of Infectious Diseases and Pneumophthisiology Craiova and Pneumology Hospital Leamna, Dolj County, diagnosed through both laboratory methods (sputum-smear positivity, gene Xpert) and radiologic examinations, were recruited for the prospective study, after obtaining written informed consent. Patients were included in our study if they had not received more than one week of anti-TB treatment. Patients with extra-pulmonary TB and co-morbidities such as HIV, diabetes, hypertension, hepatic or renal disorders, cancer, thyroid and parathyroid gland dysfunctionalities were excluded, after examination of patient history and baseline laboratory investigations. Patients treated with immunosuppressants, corticosteroids, thiazide diuretics, other drugs that may interfere with VD levels (some anticonvulsants, theophylline) and pregnant women were also excluded from the study. We also excluded those who were administered supplements based on VD.

For 17 patients it was possible to quantify all the parameters at the inclusion of the study (T0) and after they received for two months (T2) a different regimen of first-line anti-TB drugs (isoniazid, rifampicin, pyrazinamide, ethambutol). It is important to underline that none of the patients received VD supplements or any other medication that could interfere with VD plasmatic levels from T0 to T2.

Sample collection and handling. At every visit, three samples of blood were obtained, using heparinized vacutainers, with EDTA and without anticoagulant, and further centrifuged at $1,000 \mathrm{x} \mathrm{g}$, within $30 \mathrm{~min}$ of collection. Plasma/serum separated were aliquoted (minimum $500 \mu \mathrm{l}$ ) and stored at $-80^{\circ} \mathrm{C}$ until biomarker analysis.

Laboratory assessments. Assessment of the serum 25-(OH)-D level, the recommended analyte for evaluation of VD status, was performed at the Clinical Laboratory of the Clinical Emergency Hospital of Craiova on the platform Roche Cobas e601 by electrochemiluminescence immunoassay using Roche Cobas Vitamin D total reagent (Roche Diagnostics $\mathrm{GmbH})$. VDR, M30, LL-37 and beclin-1 were assayed at the Biochemistry Laboratory of the University of Medicine and Pharmacy of Craiova using commercially available kits based on enzyme immunoassays. Cusabio Human Vitamin D Receptor ELISA kit (CUSABIO) was used for VDR dosage following the steps recommended by the manufacturer. M30 Apoptosense ELISA (PEVIVA) kit (TECOmedical AG), a one-step solid-phase in vitro immunologic assay, was used for dosage of serum M30 as it measures levels of soluble caspase-cleaved K18 fragments. Both ELISA assays for LL-37 (Human LL-37 ELISA kit, Elabscience Biotechnology) and beclin-1 (Human Beclin-1 ELISA kit, Cloud-Clone Corp.), with excellent sensitivity and specificity, were carried out according to the manufacturer's protocols. All enzyme immunoassays were performed in duplicate using a complete StatFax ELISA line (orbital shaker, washing system, plate reader) provided by Awareness Technology Inc.

Statistical analysis. In the statistical analysis, nonparametric data (small sample size) are presented as the median \pm interquartile range (IQR). Qualitative variables (sex, environment, culture) are presented as percentages. The nonparametric Wilcoxon matched-pairs signed rank test was used to evaluate the significant differences between variables at T0 (before anti-TB treatment) and T2 (after two months of anti-TB treatment). Patients were divided into two groups according to the results obtained at culture analysis (positive or negative) after two months of treatment. The two groups were compared using the nonparametric Mann-Whitney U test. Differences were considered statistically significant at the $5 \%$ level (two-tailed). The strength of quantitative relationship 
between different clinical characteristics of the patients was measured with Spearman coefficients and heatmap. Data were analyzed using GraphPad Prism 8.4.3 software (GraphPad Software, LLC).

\section{Results}

We recruited 30 patients but only 17 were analyzed during the period of this study. Their mean age \pm standard deviation (SD) was $48.76 \pm 8.83$ (range: $32-61$ years) and weight was 53.88 \pm 7.77 (range: $39-64 \mathrm{~kg}$ ). Demographic characteristics are presented in Table I and biochemical characteristics are presented in Table II.

Serum VD levels were significantly different between the two periods of time (before and after two months of anti-TB treatment) being lower at T0 than at T2 (Fig. 1). After two months of first-line anti-TB treatment, patients were divided into two different groups according to the results obtained after sputum-culture analysis; 6 patients were still positive and had bacillary load at T2 and 11 patients were negative at T2. In patients with positive sputum-culture, the serum levels of VD and VDR varied differently (3 patients presented lower VDR levels at T2, while VD levels increased, 2 patients presented higher VDR levels at T2, while VD levels decreased and only 1 patient was identified with higher values for both VD and VDR).

A negative moderate correlation was found between sex and VD level (rho=-0.58, $\mathrm{P}=0.032$ ), VD decreasing more for female patients (Table III). No significant correlations were found between serum levels of the biomarkers (Fig. 2).

No significant differences in VD, VDR, LL-37, beclin-1 or M30 were noted among the TB-positive culture and TB-negative culture group after two months of treatment (Table IV). The results revealed decreased values in negative patients compared with positive TB patients, except for LL-37, but the differences were not significant (Fig. 3).

\section{Discussion}

All the enrolled patients included in the present study were diagnosed with primary active pulmonary tuberculosis (TB) through acid-fast bacilli microscopic identification and radiologic chest examinations at $\mathrm{T} 0$ and administration of first-line specific anti-TB treatment (isoniazid, rifampicin, pyrazinamide, ethambutol) was initiated at a weight-dependent dosage. Pyridoxine (vitamin B6) was the only supplement administered during the therapy.

The risk of developing infectious pulmonary diseases, including $\mathrm{TB}$, is related to malnutrition, tobacco and alcohol consumption, and low socioeconomic status (14). In line with this, all the included patients presented at the hospitals were active smokers or alcohol consumers, with malnutrition and $76.5 \%$ came from rural environment. Moreover, our analysis showed vitamin $\mathrm{D}_{3}$ (VD) deficiency before anti-TB treatment (T0) as a feature of all patients with pulmonary TB included in our study, suggesting impaired antimycobacterial innate immunity mechanisms, in congruence with other studies (14-16) which report that the VD axis plays an essential role in innate defense against intracellular microorganisms.
Table I. Demographic characteristics of the TB patients $(\mathrm{N}=17)$ included in this study.

\begin{tabular}{lc}
\hline Characteristics & Data \\
\hline Age, median (IQR) & $51(43-55)$ \\
Weight, median (IQR) & $58(47.5-59.5)$ \\
Sex, n (\%) & \\
Female & $3(17.7)$ \\
Male & $14(82.3)$ \\
Environment, n (\%) & \\
Urban & $4(23.5)$ \\
Rural & $13(76.5)$ \\
\hline
\end{tabular}

TB, tuberculosis; IQR, interquartile range.

It is important to emphasize that both VD and the vitaminD receptor (VDR) increased after two months of first-line anti-TB pharmacotherapy without administering other drugs interfering with VD serum levels or nutritive supplements. Increased serum VD levels as a treatment outcome support the antimycobacterial effects of anti-TB treatment and a higher sputum conversion rate (14).

Macrophages recognize mycobacteria through specific receptors, Toll-like receptors (TLRs) and further enhance VDR expression $(15,16)$. VDR promotes cathelicidin (LL-37) synthesis and activity, leading to bacterial membrane disintegration (15). In our study, $81.81 \%$ of the patients (9 patients) with negative sputum-culture at T2 presented proportionally increased serum levels for both VD and VDR, while only $18.18 \%$ (2 patients who initially presented higher bacillary loads at $\mathrm{T} 0$ ) were identified with lower levels of VDR and higher levels of VD. Decreased levels of VDR may be due to downregulation of its expression. In addition, VDR gene polymorphisms may influence VDR activity and downstream VD-mediated effects (15-18). Lack of VDR could suggest that serum VD are not sufficient to promote innate immune responses (15-18). Further studies regarding VD supplementation in pulmonary TB patients may be useful in establishing the importance of this liposoluble vitamin in immunity and antimycobacterial processes, as available clinical trials have given inconclusive results to date (18).

VD-mediated innate immunity and autophagy have been shown to provide protection against infection with Mycobacterium tuberculosis (M.tb) (14-17). After VD binding to VDR through VD response elements and induction of oxidative stress burst, LL-37 expression is increased and autophagy is upregulated through phagosomal maturation $(14,18)$. Elimination of M.tb. is dependent on both LL-37 and beclin-1, as higher levels determine better intracellular bacterial killing $(1,6)$. Several studies have pointed out that not only susceptibility to infection, but also impaired expression of LL-37 has been associated with VD deficiency, as this antimicrobial peptide is highly dependent on VD concentration (18-20). As Ayelign et al emphasize, lower VD levels are connected with lower antimicrobial LL-37 influence and delayed autophagy mechanisms (16). 
Table II. Biochemical characteristics of the TB patients $(\mathrm{N}=17)$ included in this study.

\begin{tabular}{lccc}
\hline Biochemical parameters & T0 median $(\mathrm{IQR})$ & T2 median (IQR) & P-value \\
\hline VDR & $169(124.5-290.3)$ & $242.8(149.5-340.9)$ & 0.1439 \\
VD & $9.1(5.5-16)$ & $14.4(8-22.6)$ & $0.0181^{\mathrm{a}}$ \\
LL-37 & $25.6(13.75-76.25)$ & $26.1(12.9-51.88)$ & 0.6322 \\
Beclin-1 & $0.21(0.145-0.25)$ & $0.13(0.08-0.30)$ & 0.3755 \\
M30 & $202.2(113.1-289.7)$ & $151.3(133.2-264.4)$ & 0.8603 \\
\hline
\end{tabular}

TB, tuberculosis; T0, before antituberculosis treatment; T2, after two months of antituberculosis treatment; VDR, vitamin D receptor; $\mathrm{VD}$, vitamin $\mathrm{D}_{3}$; LL-37, cathelicidin; $\mathrm{M} 30$, apoptosis biomarker; IQR, interquartile range. All data are presented as the median (IQR). ${ }^{\mathrm{a}}<0.05$; significant $\mathrm{P}$-value among groups; the Mann-Whitney $\mathrm{U}$ test is used for the two groups.

Table III. Correlations between biochemical and demographic characteristics of the TB patients (Spearman coefficients with correlation matrix).

\begin{tabular}{|c|c|c|c|c|c|c|c|c|c|}
\hline & 1 & 2 & 3 & 4 & 5 & 6 & 7 & 8 & 9 \\
\hline Age (1) & 1.00 & & & & & & & & \\
\hline $\operatorname{Sex}(2)$ & 0.13 & 1.00 & & & & & & & \\
\hline Weight (3) & 0.00 & $-0.67^{b}$ & 0.00 & & 1 & & & & \\
\hline Environment (4) & -0.07 & -0.11 & -0.01 & 1.00 & & & & & \\
\hline VDR_T2 (5) & 0.28 & -0.05 & 0.17 & 0.19 & 1.00 & & & & \\
\hline VD_T2 (6) & -0.19 & $-0.58^{\mathrm{a}}$ & 0.06 & 0.03 & 0.18 & 1.00 & & & \\
\hline LL-37_T2 (7) & 0.05 & -0.02 & 0.10 & -0.16 & 0.13 & 0.49 & 1.00 & & \\
\hline M30_T2 (8) & 0.25 & -0.30 & 0.48 & 0.00 & -0.16 & 0.07 & 0.44 & 1.00 & \\
\hline Beclin-1_T2 (9) & -0.22 & -0.47 & 0.37 & -0.25 & -0.21 & 0.03 & -0.23 & 0.30 & 1.00 \\
\hline
\end{tabular}

${ }^{\text {a }} \mathrm{P}<0.05 ;{ }^{\text {b }}<0.01$. TB, tuberculosis; $\mathrm{T} 2$, after two months of antituberculosis treatment; VDR, vitamin D receptor; VD, vitamin $\mathrm{D}_{3}$; LL-37, cathelicidin; M30, apoptosis biomarker.

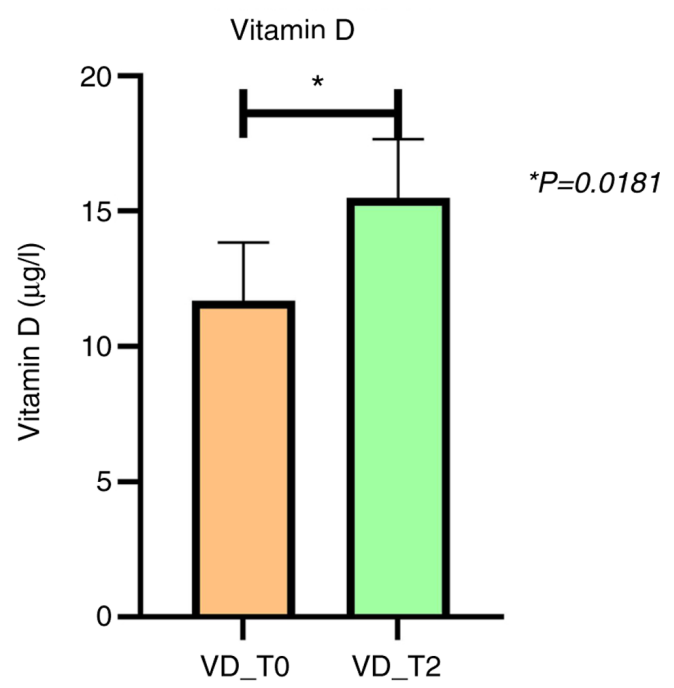

Figure 1. Bar plot of vitamin $\mathrm{D}_{3}(\mathrm{VD})$ levels before (T0) and 2 months after TB treatment (T2). Data are expressed as the mean \pm SEM.

The present study reported decreased values of LL-37, beclin-1 and M30 at T2 in culture-negative patients compared with culture-positive patients. Culture-positive individuals

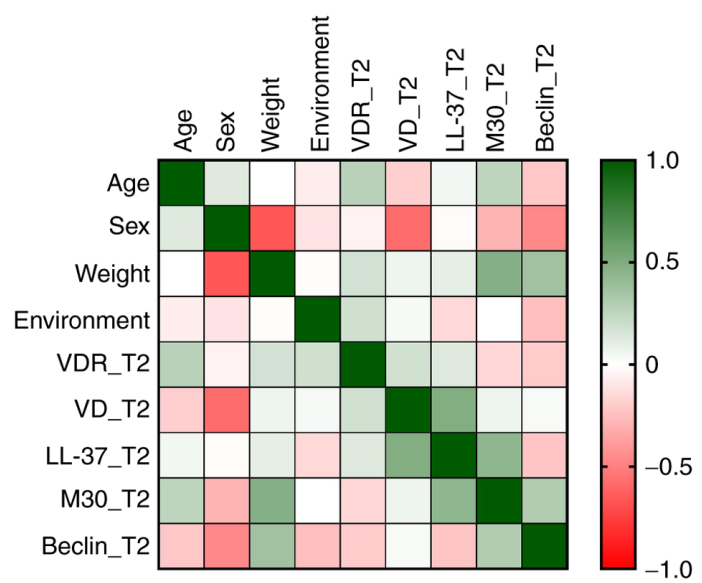

Figure 2. Heatmap of the correlation matrix between serum biomarkers and demographic characteristics of the TB patients. TB, tuberculosis; T2, after two months of antituberculosis treatment; VDR, vitamin D receptor; VD, vitamin $\mathrm{D}_{3}$; LL-37, cathelicidin; M30, apoptosis biomarker.

presented activated immunological pathways and signaling as an attempt to maintain intracellular homeostasis and restriction of M.tb. growth (6). VD actively increases LL-37 expression and promotes pathogen clearance by recruiting 
Table IV. Biomarkers after two months of anti-TB treatment.

\begin{tabular}{|c|c|c|c|}
\hline $\begin{array}{l}\text { Biomarkers Mean }( \pm \mathrm{SD}) \\
\text { Median }(\mathrm{IQR})\end{array}$ & TB-positive culture group $(n=6)$ & TB-negative-culture group $(\mathrm{n}=11)$ & P-value \\
\hline VD & $\begin{array}{l}17.96( \pm 9.29) \\
14.40(11.75-25.94)\end{array}$ & $\begin{array}{l}14.27( \pm 8.12) \\
11.7(6.51-23.18)\end{array}$ & 0.4938 \\
\hline VDR & $\begin{array}{l}356( \pm 304.2) \\
236(130.1-608.1)\end{array}$ & $\begin{array}{l}247.7( \pm 85.67) \\
248.5(163.5-332.6)\end{array}$ & 0.4923 \\
\hline LL-37 & $\begin{aligned} 35.73 & ( \pm 17.29) \\
42 & (17.73-49.03)\end{aligned}$ & $\begin{array}{l}41.55( \pm 39.33) \\
21.8(10.98-91.65)\end{array}$ & 0.8131 \\
\hline Beclin-1 & $\begin{array}{l}0.23( \pm 0.16) \\
0.20(0.07-0.39)\end{array}$ & $\begin{array}{l}0.18( \pm 0.15) \\
0.10(0.09-0.23)\end{array}$ & 0.7112 \\
\hline M30 & $\begin{array}{l}241.4( \pm 162.9) \\
187.9(130-339.9)\end{array}$ & $\begin{array}{l}229.9( \pm 177.6) \\
151.3(123.9-319.2)\end{array}$ & 0.7925 \\
\hline
\end{tabular}

$\mathrm{TB}$, tuberculosis; IQR, interquartile range; VDR, vitamin D receptor; VD, vitamin $\mathrm{D}_{3}$; LL-37, cathelicidin; M30, apoptosis biomarker.

A VD positive vs. negative

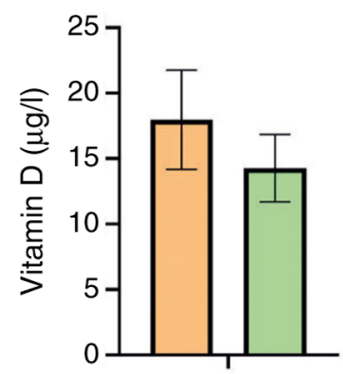

B VDR positive vs. negative

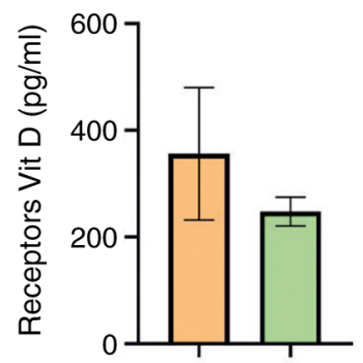

C Beclin-1 positive vs. negative

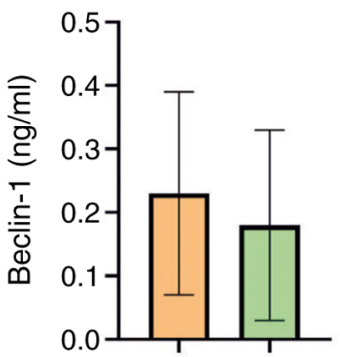

D Cathelicidin positive vs. negative

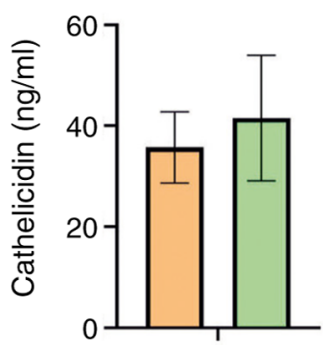

口 Positive

ㅁ Negative

E M30 positive vs. negative

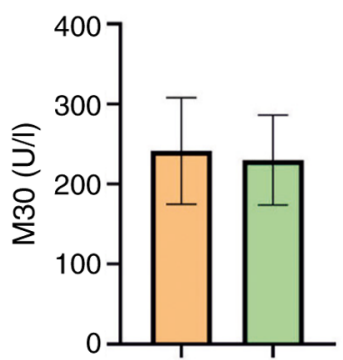

Figure 3. Values of biomarkers [(A) VD, (B) VDR, (C) Beclin-1, (D) cathelicidin, (E) M30] in positive vs. negative culture patients after two months of anti-TB treatment (mean $\pm \mathrm{SEM})$. VDR, vitamin $\mathrm{D}$ receptor; $\mathrm{VD}$, vitamin $\mathrm{D}_{3} ; \mathrm{M} 30$, apoptosis biomarker.

$\mathrm{T}$ cells and enhancing immunomodulatory activities, whereas the mycobacteria downregulate it and delay disruption of physical microbial membrane integrity through TLR signaling $(8,17,18,21)$. Research on cell culture has confirmed that inhibition of M.tb. development was sustained by both LL-37 upregulation and VD supplementation (18).

M.tb. adopts different strategies in order to evade immune macrophagic mechanisms, by restraining phago-lysosomal fusion, increasing secretion of pro-inflammatory biomarkers and generating necrosis instead of apoptosis $(15,18)$. Necrosis determines irreversible membrane and unbeneficial host-cellular destruction, while apoptosis restores normal cellular functionality as it attenuates accumulation of misfolded peptides in endoplasmic reticulum, therefore limiting TB progression (10). Caseous granuloma formation, tissue remodelling and cavitation result as a consequence of M.tb.-induced necrosis (10). On the other hand, macrophages and dendritic cells are responsible for apoptotic body degradation, enhancing both innate and adaptive responses (10). Along with LL-37 and beclin-1, M30 could also be a useful clinical marker for disease assessment and prognosis (11), as it contributes to visualize structural cellular changes and to understand different pathophysiological mechanisms involved in TB.

Our present study presented several limitations. The small number of included patients was due to the pandemic 
period of COVID-19, as access to hospitals from our town was restricted. We assessed biomarkers only after two months of anti-TB therapy but a longer period would have been useful to examine the influence of treatment on immune mechanisms such as autophagy and apoptosis. Therefore, we intend to perform further studies with more clinical cases in the future. In the present study, we recruited only patients who had not received VD supplements or drugs interfering with VD metabolic activity, but we did not monitor food intake, sunlight exposure or genetic polymorphisms of VDR, which can also affect the risk of TB activation and progression (14). With regard to the demographics, most of our patients showed a male predominance and this may be a potential bias.

Nevertheless, one of the major strengths of our study is represented by the analysis of M30 involvement in pulmonary $\mathrm{TB}$, as the first so far to the best of our knowledge. The unique cohort of subjects, without comorbidities and only pulmonary infection with mycobacteria, strongly indicates the value of our correlations.

All in all, both autophagy and apoptosis biomarkers as well as the VD axis and immunological response variations in pulmonary TB should not be neglected in further studies, as they may be useful in controlling this highly infectious public threat.

\section{Acknowledgements}

We are thankful for the technical support provided by chemist Loredana Colhon from the Department of Biochemistry, University of Medicine and Pharmacy of Craiova, Romania, and to all the patients who gave consent for participating in the present study.

\section{Funding}

No funding was received.

\section{Availability of data and materials}

The datasets used and/or analysed during the current study are available from the corresponding author on reasonable request.

\section{Authors' contributions}

ADM, SS, MB and ATS were involved in the literature research and wrote the manuscript. ATS supported the statistical analysis and reviewed the results. CGP, ADM and SS conceived, planned and followed the execution of the experiments. CGP and AMB administered the 25-OH-D dosage and contributed to the manuscript revision. FMN, RC and MM collected and analyzed the patient samples. All authors contributed to the manuscript revision, read and approved the final version.

\section{Ethics approval and consent to participate}

The study was approved by The Ethics Committees of The University of Medicine and Pharmacy of Craiova, Romania (Nr. 5/17.01.2019). All patients included in the study provided informed consent for data publication.

\section{Patient consent for publication}

Not applicable.

\section{Competing interests}

The authors declare that they have no competing interests.

\section{Authors' information}

This study is part of the Ph.D. thesis of Andreea-Daniela Meca from the University of Medicine and Pharmacy of Craiova, Romania.

\section{References}

1. Rekha RS, Mily A, Sultana T, Haq A, Ahmed S, Mostafa Kamal SM, van Schadewjik A, Hiemstra PS, Gudmundsson GH, Agerberth B and Raqib R: Immune responses in the treatment of drug-sensitive pulmonary tuberculosis with phenylbutyrate and vitamin $\mathrm{D}_{3}$ as host directed therapy. BMC Infect Dis 18: 303, 2018.

2. Geneva, World Health Organization: WHO Global Tuberculosis Report. Geneva World Health Organization, pp1-261, 2019. https://www.who.int/tb/publications/global report/en/.

3. WHO Regional Office for Europe, European Centre for Disease Prevention and Control. Tuberculosis Surveillance and Monitoring in Europe 2018-2016 Data. World Health Organization, pp181-182, 2018. https://www.ecdc.europa. $\mathrm{eu} / \mathrm{en} /$ publications-data/tuberculosis-surveillance-and-monitoring-europe-2018.

4. Ashenafi S, Mazurek J, Rehn A, Lemma B, Aderaye G, Bekele A, Assefa G, Chanyalew M, Aseffa A, Andersson J, et al: Vitamin $\mathrm{D}_{3}$ status and the association with human cathelicidin expression in patients with different clinical forms of active tuberculosis. Nutrients 10: 721, 2018

5. Jo EK: Autophagy as an innate defense against mycobacteria. Pathog Dis 67: 108-118, 2013.

6. Paik S, Kim JK, Chung C and Jo EK: Autophagy: A new strategy for host-directed therapy of tuberculosis. Virulence 10: 448-459, 2019.

7. Balcells ME, Yokobori N, Hong BY, Corbett J and Cervantes J: The lung microbiome, vitamin $\mathrm{D}$, and the tuberculous granuloma: A balance triangle. Microb Pathog 131: 158-163, 2019.

8. Torres-Juarez F, Cardenas-Vargas A, Montoya-Rosales A, González-Curiel I, Garcia-Hernandez MH, Enciso-Morenzo JA, Hancock RE and Rivas-Santiago B: LL-37 immunomodulatory activity during Mycobacterium tuberculosis infection in macrophages. Infect Immun 83: 4495-4503, 2015.

9. Gupta S, Winglee K, Gallo R and Bishai WR: Bacterial subversion of cAMP signalling inhibits cathelicidin expression, which is required for innate resistance to Mycobacterium tuberculosis. J Pathol 242: 52-61, 2017.

10. Lam A, Prabhu R, Gross CM, Riesenberg LA, Singh V and Addarwal S: Role of apoptosis and autophagy in tuberculosis. Am J Physiol Lung Cell Mol Physiol 313: L218-L229, 2017.

11. Lee KS, Chung JY, Jung YJ, Chung WY, Park JH, Sheen SS, Lee KB and Park KJ: The significance of caspase-cleaved cytokeratin 18 in pleural effusion. Tuberc Respir Dis (Seoul) 76: 15-22, 2014.

12. Mohareer K, Asalla S and Banerjee S: Cell death at the cross roads of host-pathogen interaction in Mycobacterium tuberculosis infection. Tuberculosis (Edinb) 113: 99-121, 2018.

13. Elliott TO, Owolabi O, Donkor S, Kampmann B, Hill PC, Ottenhoff TH, Haks MC, Kaufmann SH, Maertzdorf J and Sutherland JS: Dysregulation of apoptosis is a risk factor for Tuberculosis disease progression. J Infect Dis 212: 1469-1479, 2015.

14. Wang M, Kong W, He B, Li Z, Song H, Shi P and Wang J: Vitamin $\mathrm{D}$ and the promoter methylation of its metabolic pathway genes in association with the risk and prognosis of tuberculosis. Clin Epigenetics 10: 118, 2018.

15. Chung C, Silwal P, Kim I, Modlin RL and Jo EK: Vitamin Dcathelicidin axis: At the crossroads between protective immunity and pathological inflammation during infection. Immune Netw 20: e12, 2020. 
16. Ayelign B, Workneh M, Molla MD and Dessie G: Role of vitamin-D supplementation in TB/HIV co-infected patients Infect Drug Resist 13: 111-118, 2020.

17. Alford MA, Baquir B, Santana FL, Haney EF and Hancock RE Cathelicidin host defense peptides and inflammatory signaling: Striking a balance. Front Microbiol 11: 1902, 2020.

18. Rode AKO, Kongsbak M, Hansen MM, Lopez DV, Levring TB Woetmann A, Ødum N, Bonefeld CM and Geisler C: Vitamin D counteracts Mycobacterium tuberculosis-induced cathelicidin downregulation in dendritic cells and allows Th1 differentiation and IFN $\gamma$ secretion. Front Immunol 8: 656, 2017.

19. Xia J, Shi L, Zhao L and Xu F: Impact of vitamin D supplementation on the outcome of tuberculosis treatment: A systematic review and meta-analysis of randomized controlled trials. Chin Med J (Engl) 127: 3127-3134, 2014.
20. Talat N, Perry S, Parsonnet J, Dawood G and Hussain R: Vitamin D deficiency and tuberculosis progression. Emerg Infect Dis 16: 853-855, 2010

21. Rajamanickam A, Munisankar S, Dolla CK and Babu S: Diminished systemic and mycobacterial antigen specific anti-microbial peptide responses in low body mass index-latent tuberculosis co-morbidity. Front Cell Infect Microbiol 10: 165, 2020.

(i) $\odot$ This work is licensed under a Creative Commons Attribution-NonCommercial-NoDerivatives 4.0 International (CC BY-NC-ND 4.0) License. 